\title{
Estimation of turbulence dissipation rate by Large eddy PIV method in an agitated vessel
}

\author{
Bohuš Kysela $^{1 \text { a }}$, Darina Jašíková ${ }^{2}$, Jiř́i Konfršt ${ }^{1}$, Radek Šulc ${ }^{3}$, and Pavel Ditl ${ }^{3}$ \\ 1 Institute of Hydrodynamics, AS CR, v.v.i. \\ Pod Patankou 30/5, 16612 Prague 6, Czech Republic \\ 2 Institute for Nanomaterials, Advanced Technology and Innovation, Technical University of Liberec, \\ Studentska 1402/2, 46117 Liberec, Czech Republic \\ 3 Department of Process Engineering, Faculty of Mechanical Enginnering, Czech Technical University in Prague, \\ Technicka 4, 16607 Prague 6, Czech Republic.
}

\begin{abstract}
The distribution of turbulent kinetic energy dissipation rate is important for design of mixing apparatuses in chemical industry. Generally used experimental methods of velocity measurements for measurement in complex geometries of an agitated vessel disallow measurement in resolution of small scales close to turbulence dissipation ones. Therefore, Particle image velocity (PIV) measurement method improved by large eddy PIV approach was used. Large eddy PIV method is based on modeling of smallest eddies by a sub grid scale (SGS) model. This method is similar to numerical calculations using Large Eddy Simulation (LES) and the same SGS models are used. In this work the basic Smagorinsky model was employed and compared with power law approximation. Time resolved PIV data were processed by Large Eddy PIV approach and the obtained results of turbulent kinetic dissipation rate were compared in selected points for several operating conditions (impeller speed, operating liquid viscosity).
\end{abstract}

\section{Introduction}

Distribution of the turbulence kinetic energy dissipation rate inside an agitated vessel is crucial for better understanding of mixing processes such as dispergation, homogenization etc. and also essential for scale-up modelling of large mixing vessels. The direct measurement of distribution of turbulent kinetic energy dissipation rate is not available, therefore the determination is based on background of turbulence theories [1,2]. Several approaches how to determine the dissipation rate in mixing vessel were presented by Kresta at al. [3], thereafter many other researchers have attempted to improve the proposed methods e.g. [4-12], but the results of absolute values of local dissipation rates have not been accomplished yet. While formerly a temporal record of instantaneous velocities [3, 12] or two point measurement [9] was used for determination of dissipation rate, nowadays determination from velocity field obtained by PIV method $[5,6,8,10,11]$ is preferred. This trend will be probably continued according to measurement techniques development to fully 3D measurements with resolution up to Kolmogorov scales. Meanwhile we want to investigate and make clear the relation between distribution of dissipation rate inside an agitated vessel and operating conditions for high impeller Reynolds numbers (turbulent regime).

\subsection{Turbulence energy dissipation rate estimation from PIV measurements}

The determination of the turbulent kinetic energy dissipation directly from PIV (Particle Image Velocimetry) measurements by calculations of fluctuating strain rate tensor was analysed e.g. in [13]. The local mean dissipation rate $\epsilon$ was calculated from approximation

$$
\epsilon=2 v\left\langle s_{i j} s_{i j}\right\rangle=v \overline{\frac{\partial u_{i}}{\partial x_{j}}\left(\frac{\partial u_{i}}{\partial x_{j}}+\frac{\partial u_{j}}{\partial x_{i}}\right)},
$$

where $v$ is kinematic viscosity, $s_{i j}$ fluctuating strain rate tensor, $u_{i}$ is ith component of fluctuating velocity and brackets \langle\rangle or over-line represents time-average. The direct estimation from the 2D PIV measurements has two limiting factors the first one is the measurement of two velocity components and the second is the temporal and spatial resolution.

Only two components of radial $u$ and axial $v$ velocity (referred as $x$ and $y$ direction respectively) are obtained by measurement, then all components of the strain rate tensor are not known. Hence, the missing components are necessary to substitute with some presumptions. The first one is presumption of turbulent isotropic flow. We know, that this presumption is not quite valid and the equation for two velocity components is used to show a comparison with values based on dominant convective velocity in axial $(y)$ direction.

$$
\epsilon=6 v\left(\overline{\left(\frac{\partial v}{\partial x}\right)^{2}}+\overline{\left(\frac{\partial v}{\partial y}\right)^{2}}+\overline{\frac{\partial u}{\partial y} \cdot \frac{\partial v}{\partial x}}\right)
$$

${ }^{a}$ e-mail: kysela@ih.cas.cz 
The anisotropy of the flow was solved differently by various authors. Sheng at al. [5] showed that the known components of dissipation tensor have similar distribution and magnitude and assumed that the dissipation rate can be approximated by multiplying the sum of the known components by factor of $9 / 5$.

$\epsilon=v \frac{9}{5}\left(2 \overline{\left(\frac{\partial u}{\partial x}\right)^{2}}+2 \overline{\left(\frac{\partial v}{\partial y}\right)^{2}}+\overline{\left(\frac{\partial u}{\partial y}\right)^{2}}+\overline{\left(\frac{\partial v}{\partial x}\right)^{2}}+2 \overline{\frac{\partial u}{\partial y} \cdot \frac{\partial v}{\partial x}}\right)$

It is also necessary to solve the effect of spatial resolution on dissipation values. Saarenrine and Piirto [13] performed measurements in channel with backward-facing step and compared several cases with different image areas and with different spatial resolution. They concluded that the best estimate for dissipation rate is achieved when spatial resolution of the PIV systems is close to the Kolmogorov length scale $\eta$ (equation4) and the velocity error is kept small. For flows with high dissipation rate values and small Kolmogorov length scales it is very difficult to get reliable results. Unfortunately, the limitations and the achieved accuracy depend on the flow case.

$$
\eta=\left(\frac{v^{3}}{\epsilon}\right)^{1 / 4}
$$

The mean value of Kolmogorov length scale in agitated vessel can be determined from the mean dissipation rate $\bar{\epsilon}$ calculated (for $H=T, D=T / 3$ ) by relation:

$$
\bar{\epsilon}=\frac{P}{V \rho}=\frac{4}{27 \pi} \operatorname{Pon}^{3} D^{2},
$$

where $P$ is power input, $V$ agitated vessel volume, $\rho$ operating liquid density, $P o$ impeller power number, $n$ impeller speed, $D$ impeller diameter. Then the mean Kolmogorov length scale is $\eta \approx 37 \mu m$ for vessel diameter $D=133 \mathrm{~mm}$, impeller speed $n=300 \mathrm{rpm}$, kinematic viscosity $v=1 \cdot 10^{-6} \mathrm{~m}^{2} \cdot \mathrm{s}^{-1}\left(\operatorname{Re}_{M}=8.8 \cdot 10^{4}\right)$ and impeller power number $P o=5.2$ (Rushton turbine impeller). Moreover, the Kolmogorov length scale in turbulent regions is much smaller. For this reason the direct estimation of turbulent kinetic energy dissipation in an agitated vessel for fully turbulent regime $\left(\operatorname{Re}_{M}>10^{4}\right)$ by common PIV measurements is unfeasible.

$$
\operatorname{Re}_{M}=\frac{n D^{2}}{v}
$$

\subsection{Large eddy PIV approach}

There is another approach used in CFD (Computational Fluid Dynamics), where the calculations are also limited by real grid size and then the SGS (sub-grid scale) models are used to resolve lower scales. This similar approach called Large eddy PIV was used for dissipation of turbulent kinetic energy in an agitated vessel e.g. by $[5,14,8,10,11]$. The basic presumption should be fulfilled: grid size has to be in inertial sub-range, where turbulent kinetic energy is transferred from large scales to small ones.

$$
\epsilon_{L E S} \approx\left\langle\epsilon_{S G S}\right\rangle=-\left\langle\tau_{i j} \bar{S}_{i j}\right\rangle,
$$

Table 1. Operating impeller Reynolds numbers for used combinations of impeller speed and used liquid viscosity.

\begin{tabular}{cccc}
\hline & water & MEG1 & MEG2 \\
\hline$v\left[m^{2} \cdot s^{-1}\right]$ & $1 \cdot 10^{-6}$ & $2 \cdot 10^{-6}$ & $3 \cdot 10^{-6}$ \\
\hline \hline $300 \mathrm{rpm}$ & 88445 & & \\
$600 \mathrm{rpm}$ & 176890 & 88445 & 58963 \\
$750 \mathrm{rpm}$ & & 110556 & 73704 \\
$850 \mathrm{rpm}$ & & & 83531 \\
\hline
\end{tabular}

where $\epsilon_{S G S}$ is energy flux at cut-off scale, $\tau_{i j}$ modelled SGS stress, $\bar{S}_{i j}$ resolved strain rate tensor. Smagorinsky eddy viscosity model is given by:

$$
\tau=-C_{s}^{2} \Delta^{2}|\bar{S}| \bar{S}_{i j},
$$

where $C_{s}=0.17$ is the Smagorinsky constant, $\Delta$ subgrid characteristic length.

$$
\epsilon_{L E S}=C_{s}^{2} \Delta^{2} \overline{\left[\frac{1}{2}\left(\frac{\partial u_{i}}{\partial x_{j}}+\frac{\partial u_{j}}{\partial x_{i}}\right)\right]^{3 / 2}} .
$$

\subsection{Power law correlation}

The influence of spatial resolution on the estimated dissipated rate can be described by the power law [10]:

$$
\epsilon_{\text {corr }}=\frac{\epsilon_{\Delta}}{(\Delta / \eta)^{-m}}
$$

where $\epsilon_{\text {corr }}$ is calculated dissipation rate for spatial resolution at Kolmogorov length scale $\eta, \epsilon_{\Delta}$ is obtained dissipation rate on measured grid, $\Delta$ is grid characteristic size. From this correlation should be also derived the Large eddy approach (with exponent equal two). We used exponent $m=1.15$ obtained by Delafosse at al. [10].

\section{Experimental}

Measurements of the velocity fields were performed in a flat bottomed mixing vessel. The vessel was equipped with four radial baffles and agitated with six-blade standard Rushton turbine (see figure 1). The stirred vessel diameter was $T=400 \mathrm{~mm}$, filled with liquid to the level $H=T$. The diameter of the impeller was $D=133 \mathrm{~mm}(T / D=3)$ and installed with off-bottom clearance $C / D=0.75$. The width of baffles was $b=0.1 T$. The measurements were carried out in turbulent regime $\left(\operatorname{Re}_{M}>10^{4}\right)$ for agitated vessel and impeller speed was chosen proportionally to Reynolds number (see table 1) 300; 600; 750; 850 rpm with combination of three working liquids, water (density $\rho=1000 \mathrm{~kg} \cdot \mathrm{m}^{-3}$, kinematic viscosity $v=1 \cdot 10^{-6} \mathrm{~m}^{2} \cdot \mathrm{s}^{-1}$ ) and two water solutions with mono-ethylene glycol MEG1 $v=2 \cdot 10^{-6} \mathrm{~m}^{2} \cdot \mathrm{s}^{-1}$ and MEG2 $v=3 \cdot 10^{-6} \mathrm{~m}^{2} \cdot \mathrm{s}^{-1}$.

The positions are presented relatively in dimensionless form, where $z^{*}=z / H$ is ratio of the total liquid depth and represents vertical position which is parallel with the axis of the impeller shaft. The axis of impeller symmetry is at $z / H=0.283$ where the central disk is. The radial position is expressed by the dimensionless radial component $r^{*}=$ $2 r / D$. 


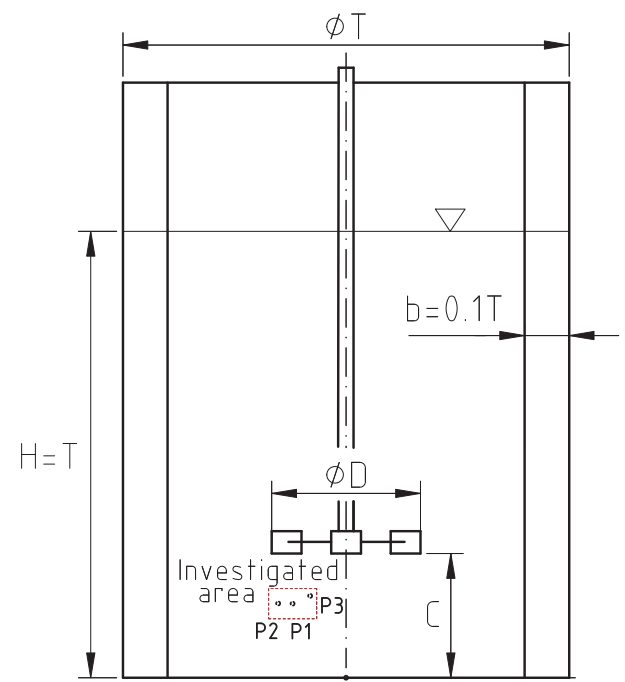

Fig. 1. The pilot plant cylindrical vessel with a six-blade standard Rushton turbine $(\mathrm{T}=400 \mathrm{~mm}, \mathrm{H} / \mathrm{T}=1, \mathrm{C} / \mathrm{D}=0.75, \mathrm{~b} / \mathrm{T}=1 / 10$, four baffles) with depicted measurement area and three analysed points.

\subsection{PIV measurement}

The results from region under the impeller were obtained by the PIV measurement technique. The investigated area was $43 \times 27 \mathrm{~mm}$ (figure 1). The area was adjusted $20.5 \mathrm{~mm}$ from the vessel axis and $31.3 \mathrm{~mm}$ below the impeller blade (figure 1). The PIV system was time-resolved LITRON LDY 304, Nd:YLF laser; high speed PIV-regime camera Speed-Sence 611 (with full resolution $1280 \times 800$ ) supplied by the DANTEC company. Rhodamine B fluorescent particles with the mean diameter $10 \mu \mathrm{m}$ were used as seeding particles along with wavelength filter to reduce laser reflections. That system was supervised by the Dynamic Studio software which was also used for velocity evaluation. The final evaluated vector grid was $79 \times 49$. The operating frequency was $1 \mathrm{kHz}$ (that means 1000 vector fields per second) the record length was $24 s$.

\section{Results}

The analysis of measured mean ensemble-averaged velocity field in selected region shows good agreement with commonly used assumptions that velocity magnitude is proportional only to impeller tip speed $v_{t i p}=\pi D n$ at fully turbulent regime $\left(\operatorname{Re}_{M}>10^{4}\right)$. Comparison of two velocity fields normalised by impeller tip speed is shown in figure 2 , where the velocity field of water with impeller speed 300 rpm is compared to the velocity field of MEG1 solution with impeller speed $600 \mathrm{rpm}$. The same figure is depicted for root mean square values of fluctuation velocity in figure 3 , where we can see that the r.m.s. values of vertical fluctuation velocity $(y)$ component is higher than horizontal component values $(x)$ which are approximately $60 \%$ of their magnitude. It indicates the anisotropy in flow which is oriented predominantly to the vertical direction. Nevertheless, both figures show good agreement in values of velocity magnitude and direction.

Then the temporal velocity data were treated and instantaneous fluctuation velocity gradients were obtained.
Table 2. Dimensionless coordinates of selected points in investigated area.

\begin{tabular}{ccc}
\hline & $\mathrm{r}^{*}$ & $\mathrm{z}^{*}$ \\
\hline P1 & 0.299 & 0.123 \\
P2 & 0.210 & 0.138 \\
P3 & 0.112 & 0.141 \\
\hline
\end{tabular}

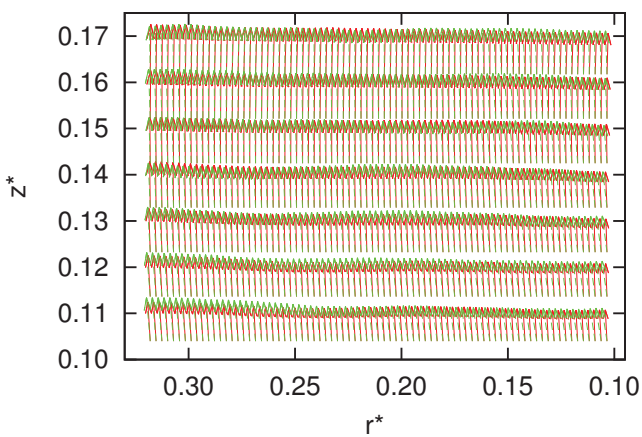

$300 \mathrm{rpm} ; v=1 \cdot 10^{-6} \mathrm{~m}^{2} \cdot \mathrm{s}^{-1} \longrightarrow$
$600 \mathrm{rpm} ; v=2 \cdot 10^{-6} \mathrm{~m}^{2} \cdot \mathrm{s}^{-1}$

Fig. 2. Mean ensemble-averaged (24 s) velocity field measured by PIV method and normalised by impeller tip speed. Comparison of water at $300 \mathrm{rpm}$ (red arrows) and MEG1 at $600 \mathrm{rpm}$ (green dash arrows).

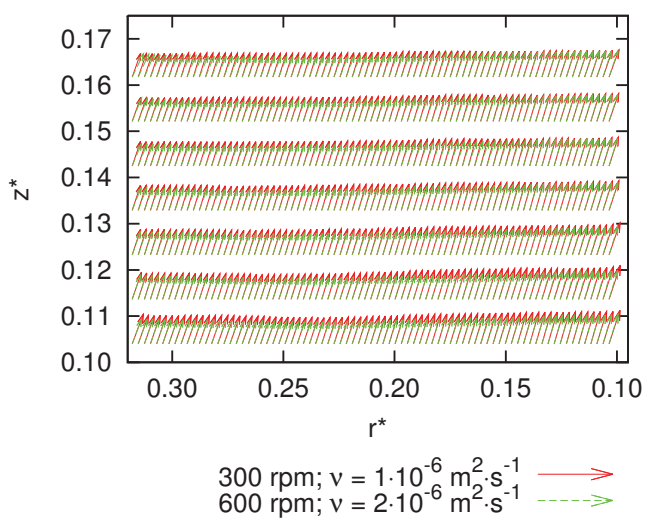

Fig. 3. Root mean square values of the fluctuation velocities (24 s) obtained from PIV measurements and normalised by impeller tip speed are depicted in vector form. Comparison of water at $300 \mathrm{rpm}$ (red arrows) and MEG1 at $600 \mathrm{rpm}$ (green dash arrows).

The derived components were introduced to equation 2 , which calculates dissipation rate based on dominant vertical velocity $(y)$ with the condition of isotropic turbulence. Identical procedure was performed for equation 3 and results were treated by power law correlation equation 10 . The comparison of the distributions of turbulent kinetic dissipation rate are depicted in figure 4 . There is visible the difference for higher values of dissipation rate which are more amplified by calculations with turbulence isotropic presumptions. The similar result was obtained for large eddy approach (figure 5 and figure 6 top). This is in agreement with the results reported in previous works e.g. [13, 14], where it was assumed that the isotropic turbulence assumption leads to an overestimation of the dissipation rate. But the main aim of our work is to investigate relation of 

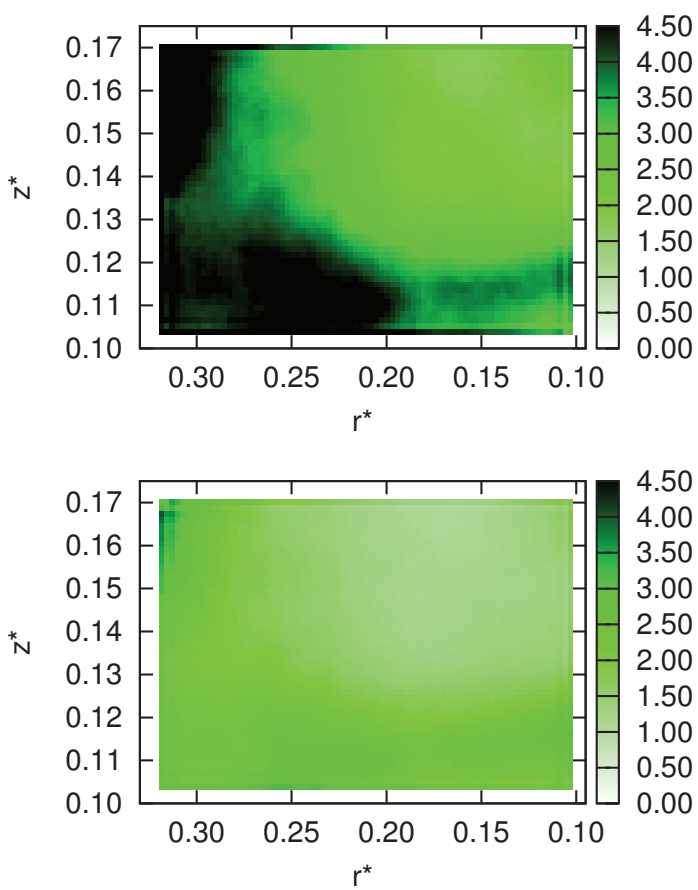

Fig. 4. Dissipation rate $\epsilon_{\text {corr }}\left[\mathrm{m}^{2} \cdot \mathrm{s}^{-3}\right]$ derived by correlation equation 10 for water and $600 \mathrm{rpm}$ with: equation 2 (top figure), and equation 3 (bottom figure).

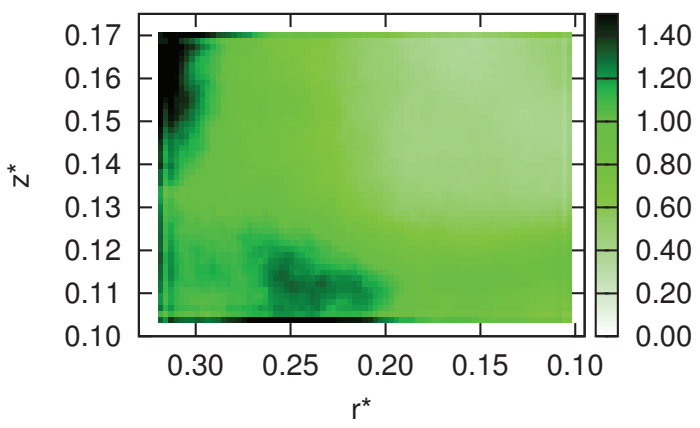

Fig. 5. Dissipation rate $\epsilon_{\text {corr }}\left[\mathrm{m}^{2} \cdot \mathrm{s}^{-3}\right]$ derived by large eddy approach equation 9 for water and $600 \mathrm{rpm}$ with equation 2 .

dissipation rate under operating conditions (impeller speed and liquid kinematic viscosity, in our case). Commonly the relation derived from integral properties based on equation 5 is used, which means that the turbulent kinetic energy dissipation rate is proportional to $n^{3} D^{2}$ and is independent of kinematic viscosity. In figure 7 are depicted distributions of dissipation rate calculated for one impeller speed $600 \mathrm{rpm}$ and three kinematic viscosities. The values of dissipation rate slightly increase with viscosity, nevertheless distribution and total magnitude is very similar, but it is very difficult to quantify the exact discrepancy in the whole field. Hence, single three points (table 2) were selected for analysis. At first the theoretical presumption of independent dimensionless dissipation rate $\epsilon / n^{3} D^{2}$ on impeller Reynolds number (in turbulent regime) was examined, but this assumption was not quite fulfilled. The dissipation rates for all impeller Reynolds numbers (table 1) are depicted in dependency: dissipation rate vs. impeller speed (figure 7 and figure 8), where the impeller speed relation $\epsilon \approx n^{3}$ is indicated. The results suggest that distribution
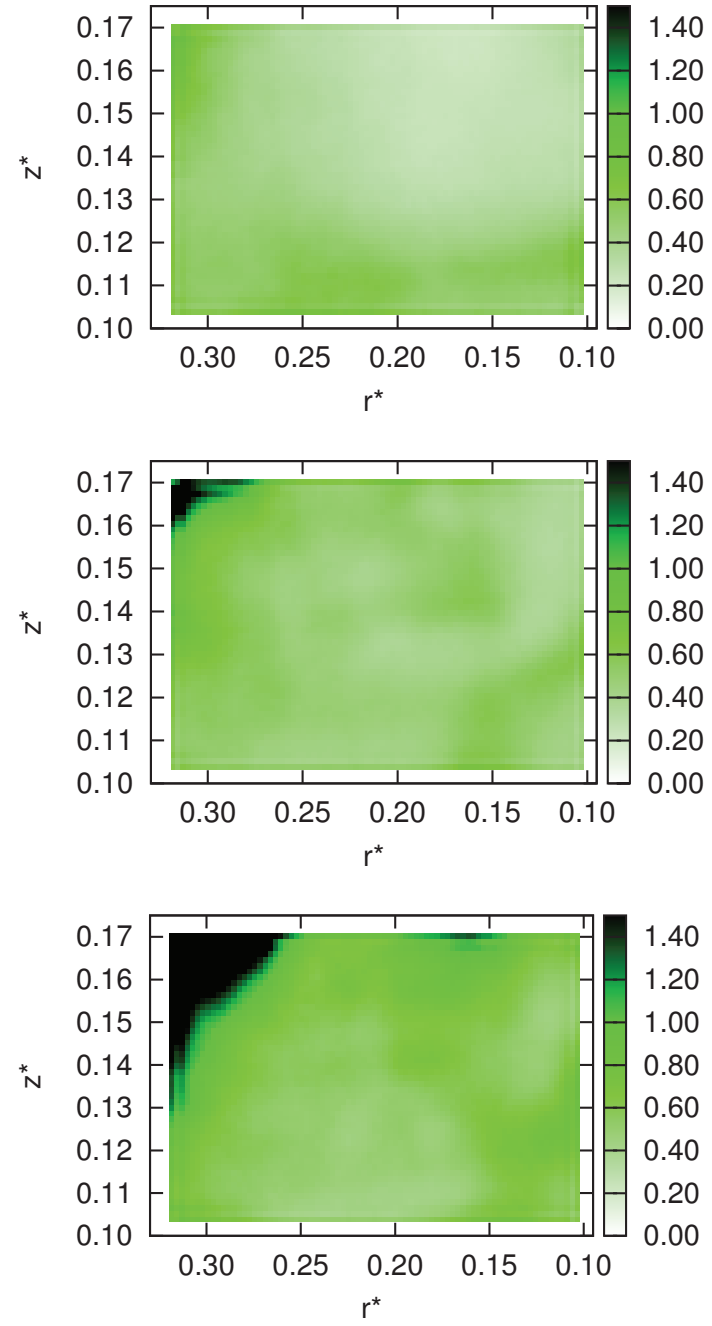

Fig. 6. Dissipation rate $\epsilon_{L E S}\left[\mathrm{~m}^{2} \cdot \mathrm{s}^{-3}\right]$ derived by large eddy approach equation 9 with equation 3 for $600 \mathrm{rpm}$ : water (top figure), MEG1 (middle figure) and MEG2 (bottom figure).

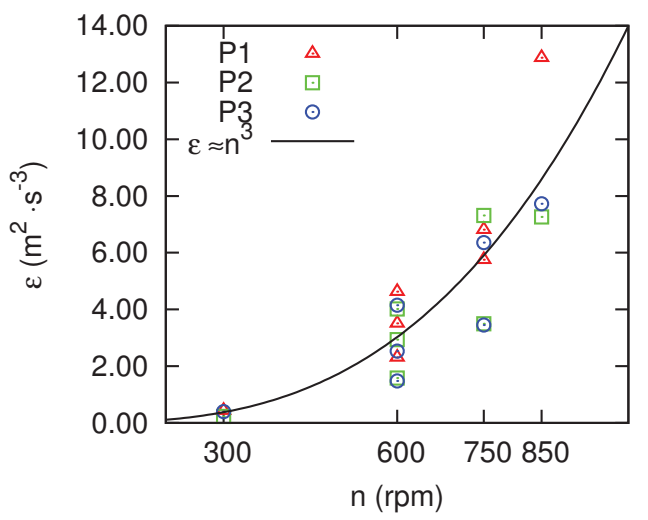

Fig. 7. Dissipation rate vs. impeller speed for all measured configurations derived by correlation equation 10 and equation 3 . 


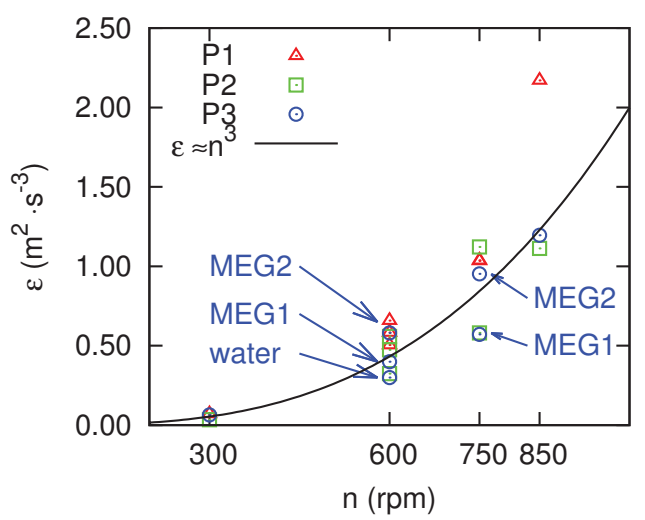

Fig. 8. Dissipation rate vs. impeller speed for all measured configurations derived by large eddy approach equation 9 and equation 3.

of dissipation rate inside agitated vessel is also dependent on liquid viscosity in regimes with high Reynolds number. The effect on viscosity is indicated in figure 8 , where the points are labeled with the type of liquid.

\section{Conclusions and discussion}

The result velocity fields obtained in agitated vessel by time resolved particle image velocimetry for three liquid viscosities and four impeller speeds were averaged by ensemble averaging. The comparison of velocity fields verified the relation of velocity field magnitude on impeller tip speed, although the discrepancy for the highest viscosity is slightly higher, probably caused by measurement accuracy. Then temporal velocity field data were treated by using large eddy approach and power law correlation to obtain the distribution of turbulent kinetic energy dissipation rate. Both proposed methods estimate similar distribution of dissipation rate, but the total magnitude is higher for power law correlation than for large eddy approach. It is also because these approaches use to many dependent constants and theoretical conditions which is difficult to fulfill, nevertheless, the results obtained by both methods indicate identical trend. The distribution of dissipation rate in agitated vessel seems to be dependent on kinematic viscosity for high impeller Reynolds numbers.

It is necessary to note, that the solution of measurement difficulties with the resolution at Kolmogorov scale and with simultaneous measurement of all three velocity components are the first step, because the estimation of real distribution of turbulent kinetic energy dissipation in an agitated vessel is more complicated. The instantaneous velocity field depends also on the position of impeller, while commonly the ensemble averaging is used, and the data are averaged during impeller movement. As well as so-called angle resolved averaging, where the data are averaged in each impeller position, it gives us a temporary discontinuous data and these data are essential and affects calculations of mean strain rate components. The best solution should be probably to measure in impeller coordinate system.

\section{Symbols}

b baffle width, $\mathrm{m}$

C off-bottom clearance, $\mathrm{m}$

D impeller diameter, $\mathrm{m}$

$\mathrm{H}$ total liquid depth, $\mathrm{m}$

$\mathrm{n} \quad$ impeller speed, $\mathrm{s}^{-1}$

$\mathrm{P} \quad$ power required by the impeller, $\mathrm{W}$

$\mathrm{P}_{\mathrm{o}} \quad$ power number, -

$\mathrm{r}^{*} \quad$ dimensionless radius of agitated vessel, -

$\mathrm{Re}_{\mathrm{M}}$ impeller Reynolds number, -

$\mathrm{T}$ vessel diameter, $\mathrm{m}$

$\mathrm{u} \quad$ r.m.s. of fluctuations velocity in $\mathrm{x}$ direction, $\mathrm{m} \cdot \mathrm{s}^{-1}$

$\mathrm{v} \quad$ r.m.s. of fluctuations velocity in y direction, $\mathrm{m} \cdot \mathrm{s}^{-1}$

$\mathrm{V}_{\text {tip }} \quad$ impeller tip speed, $\mathrm{m} \cdot \mathrm{s}^{-1}$

$\mathrm{Z}^{*} \quad$ dimensionless height of agitated vessel, -

$\epsilon \quad$ turbulent kinetic energy dissipation rate, $\mathrm{m}^{2} \cdot \mathrm{s}^{-3}$

$\bar{\epsilon} \quad$ mean energy dissipation per unit mass, $\mathrm{m}^{2} \cdot \mathrm{s}^{-3}$

$\eta \quad$ Kolmogorov length scale, $\mathrm{m}$

$v \quad$ kinematic viscosity of agitated liquid, $\mathrm{m}^{2} \cdot \mathrm{s}^{-1}$

$\rho \quad$ density of agitated liquid, $\mathrm{kg} \cdot \mathrm{m}^{-3}$

\section{Acknowledgments}

This research has been subsidized by the research project No. GA ČR P101/12/2274, RVO: 67985874 and LO1201 (National Programme for Sustainability I, MŠMT ČR).

\section{References}

1. J.O. Hinze (McGraw-Hill, 1959)

2. S.B. Pope (Cambridge University Press, 2000), ISBN 9780521598866

3. S.M. Kresta, P.E. Wood, Chemical Engineering Science 48, 1761 (1993)

4. E.S. Wernersson, C. Trägrdh, Chemical Engineering Journal 70, 37 (1998)

5. J. Sheng, H. Meng, R. Fox, Chemical Engineering Science 55, 4423 (2000)

6. R. Escudie, A. Line, AIChE Journal 49, 585 (2003)

7. S. Baldi, A. Ducci, M. Yianneskis, Chemical Engineering \& Technology 27, 275 (2004)

8. J. Kilander, A. Rasmuson, Chemical Engineering Science 60, 6844 (2005)

9. A. Ducci, M. Yianneskis, AIChE Journal 51, 2133 (2005)

10. A. Delafosse, M.L. Collignon, M. Crine, D. Toye, Chemical Engineering Science 66, 1728 (2011)

11. J. Stelmach, C. Kuncewicz, Analysys of methods for measuring energy dissipation rates in the tank with the use of a PIV system., in 14th European Conference on Mixing (2012)

12. B. Kysela, J. Konfrst, Z. Chara, LDA measurements and turbulence spectral analysis in an agitated vessel, in EFM12 - EXPERIMENTAL FLUID MECHANICS 2012, edited by Dancova, P and Novonty, P (2013), Vol. 45 of EPJ Web of Conferences, ISBN 978-807372-912-7

13. P. Saarenrinne, M. Piirto, Experiments in Fluids 29, S300 (2000), 10.1007/s003480070032

14. K.V. Sharp, R.J. Adrian, AIChE Journal 47, 766 (2001) 